\title{
INCOMPRESSIBLE MHD TURBULENCE
}

\author{
PETER GOLDREICH \\ California Institute of Technology, 150-21, Pasadena, CA 91125, USA \\ E-mail: pmg@gps.caltech.edu
}

\begin{abstract}
The inertial range of incompressible MHD turbulence is most conveniently described in terms of counter propagating waves. Shear Alfvén waves control the cascade dynamics. Slow waves play a passive role and adopt the spectrum set by the shear Alfvén waves. Cascades composed entirely of shear Alfvén waves do not generate a significant measure of slow waves. MHD turbulence is anisotropic with energy cascading more rapidly along $k_{\perp}$ than along $k_{\|}$. Anisotropy increases with $k_{\perp}$ such that the excited modes are confined inside a cone bounded by $k_{\|} \propto k_{\perp}^{2 / 3}$. The opening angle of the cone, $\theta\left(k_{\perp}\right) \propto k_{\perp}^{-1 / 3}$, defines the scale dependent anisotropy. MHD turbulence is generically strong in the sense that the waves which comprise it are critically damped. Nevertheless, deep inside the inertial range, turbulent fluctuations are small. Their energy density is less than that of the background field by a factor $\theta^{2}\left(k_{\perp}\right) \ll 1$. MHD cascades are best understood geometrically. Wave packets suffer distortions as they move along magnetic field lines perturbed by counter propagating wave packets. Field lines perturbed by unidirectional waves map planes perpendicular to the local field into each other. Shear Alfvén waves are responsible for the mapping's shear and slow waves for its dilatation. The former exceeds the latter by $\theta^{-1}\left(k_{\perp}\right) \gg 1$ which accounts for dominance of the shear Alfvén waves in controlling the cascade dynamics.
\end{abstract}

Keywords: MHD, Turbulence

\section{MHD Cascades}

A variety of models have been proposed for MHD turbulence. They share the common feature that energy cascades from lower to higher wave number.

\subsection{THE IROSNIKOV-KRAICHNAN MODEL}

The standard model is that due to Iroshnikov (1963) and Kraichnan (1965). Kraichnan's derivation of the IK spectrum relies on the fact that only oppositely directed waves interact in incompressible MHD. It assumes explicitly that the turbulence is isotropic and implicitly that the dominant interactions are those which couple 3 waves.

The above assumptions imply that the cascade time across scale $\lambda$ is

$$
t_{c} \sim\left(\frac{v_{A}}{v_{\lambda}}\right)^{2} \frac{\lambda}{v_{A}} \text {. }
$$


Setting $v_{\lambda}^{2} / t_{c}$ equal to the dissipation rate per unit mass, $\epsilon$, then yields

$$
v_{\lambda} \sim\left(\epsilon v_{A} \lambda\right)^{1 / 4},
$$

which corresponds to the $1 \mathrm{D}$ power spectrum

$$
E(k) \sim \frac{\left(\epsilon v_{A}\right)^{1 / 2}}{k^{3 / 2}} .
$$

Nonlinearity is measured by $\chi \sim\left(v_{\lambda} / v_{A}\right)$, where $N \sim \chi^{-2}$ is the number of wave periods in $t_{c}$;

$$
\chi \sim\left(\frac{\epsilon \lambda}{v_{A}^{3}}\right)^{1 / 4} .
$$

Since $\chi$ decreases with decreasing $\lambda$, only dissipation limits the length of the IK inertial range.

The IK model is flawed because the assumption of isotropy is inconsistent with the frequency and wave vector closure relations that resonant triads must satisfy (Shebalin et al., 1983). These take the form

$$
\begin{gathered}
\omega_{1}+\omega_{2}=\omega_{3}, \\
\mathbf{k}_{1}+\mathbf{k}_{2}=\mathbf{k}_{3} .
\end{gathered}
$$

But since $\omega=v_{A}\left|k_{z}\right|$, equation (5) and the $z$ component of equation (6) yield the set

$$
\begin{array}{r}
\left|k_{1 z}\right|+\left|k_{2 z}\right|=\left|k_{3 z}\right| \\
k_{1 z}+k_{2 z}=k_{3 z}
\end{array}
$$

Because nonlinear interactions can only occur between oppositely directed waves, the 3-mode coupling coefficient vanishes unless waves 1 and 2 propagate in opposite directions. In that case, equations (7) and (8) imply that either $k_{1 z}$ or $k_{2:}$ must vanish. Since one of incoming waves has zero frequency, 3-wave interactions do not cascade energy along $k_{z}$.

\subsection{INTERMEDIATE MHD TURBULENCE}

Goldreich and Sridhar $(1997)^{\star}$ propose an anisotropic MHD cascade based on scalings obtained from 3-wave interactions. It represents a new form of turbulence, which they term intermediate, because it shares some of the properties of both weak and strong turbulence. Although individual wave packets suffer small distortions in single collisions, interactions of all orders make comparable contributions to the perpendicular cascade. ${ }^{\star \star}$

* Hereafter GSII.

** This is a controversial claim. 
To derive the scaling relations for the intermediate cascade, we repeat the steps carried out in $\S 1.1$ for the IK model, but with $\lambda_{\perp}$ in place of $\lambda$ and $\lambda_{\|}$held constant. Thus

$$
t_{c} \sim\left(\frac{v_{A} \lambda_{\perp}}{v_{\lambda_{\perp}} \lambda_{\|}}\right)^{2} \frac{\lambda_{\|}}{v_{A}}
$$

Setting $\epsilon \sim v_{\lambda_{\perp}}^{2} / t_{c}$, we find

$$
v_{\lambda_{\perp}} \sim\left(\frac{\epsilon v_{\Lambda} \lambda_{\perp}^{2}}{\lambda_{\|}}\right)^{1 / 4},
$$

and

$$
E\left(k_{\perp}\right) \sim \frac{\left(\epsilon v_{A} k_{\|}\right)^{1 / 2}}{k_{\perp}^{2}} .
$$

Besides being anisotropic, the intermediate MHD cascade differs from the IK cascade in another important respect. The strength of nonlinear interactions, as measured by

$$
\chi \sim\left(\frac{v_{\lambda_{\perp}} \lambda_{\|}}{v_{A} \lambda_{\perp}}\right) \sim\left(\frac{\epsilon \lambda_{\|}^{3}}{v_{A}^{3} \lambda_{\perp}^{2}}\right)^{1 / 4},
$$

increases along the cascade. Thus, even in the absence of dissipation, the intermediate cascade has a finite inertial range. This suggests that a strong form of MHD turbulence must be the relevant one for most applications in nature.

\section{Strong MHD Turbulence}

A cascade for strong MHD turbulence is described by Goldreich and Sridhar $(1995)^{\star}$. Its defining property is that MHD waves suffer order unity distortions on time scales comparable to their periods. GSI argue that the quantity $\chi$ defined in equation (12) saturates at a value of order unity. They refer to this state as one of critical balance. Together with the assumption of a constant energy flux along the cascade, as expressed by

$$
\epsilon \sim \frac{v_{\lambda_{\perp}}^{3}}{\lambda_{\perp}}
$$

it implies that correlation lengths parallel and perpendicular to the local field direction are related by

$$
\lambda_{\|} \propto \lambda_{\perp}^{2 / 3} \text {. }
$$

* Hereafter GSI. 
Although there is a parallel cascade of energy in strong MHD turbulence, the degree of anisotropy increases along the cascade.

Let us assume $v_{L} \sim v_{A}$ and isotropy on scale outer scale $L .{ }^{\star \star}$ Then the 3dimensional energy spectrum of strong MHD turbulence takes the form

$$
E\left(k_{\perp}, k_{\|}\right) \sim \frac{v_{A}^{2}}{L^{1 / 3} k_{\perp}^{10 / 3}} f\left(\frac{k_{\|} L^{1 / 3}}{k_{\perp}^{2 / 3}}\right),
$$

where $f(u)$ is a positive symmetric function of $u$ with the properties that $f(u) \approx 1$ for $|u| \lesssim 1$ and $f(u)$ negligibly small for $|u| \gg 1$. The power spectrum is flat as a function of $k_{\|}$for $k_{\|} \lesssim k_{+}^{2 / 3} L^{-1 / 3}$ because the velocity and magnetic perturbations on transverse scale $k_{\perp}^{-\dagger}$ arise from independent wave packets whose lengths $\lambda_{\|} \sim \lambda_{\perp}^{2 / 3} L^{1 / 3}$. The 1-dimensional perpendicular power spectrum obtained from equation (15) reads

$$
E\left(k_{\perp}\right) \sim \frac{v_{A}^{2}}{L^{2 / 3} k_{\perp}^{5 / 3}} .
$$

Thus the spectrum of strong MHD turbulence is an anisotropic version of Kolmogorov's spectrum for hydrodynamic turbulence.

Inertial range velocity differences and magnetic perturbations across perpendicular scale $\lambda_{\perp}$ satisfy

$$
v_{\lambda_{\perp}} \sim b_{\lambda_{\perp}} \sim\left(\frac{\lambda_{\perp}}{L}\right)^{1 / 3} v_{A} .
$$

Thus even though the turbulence is properly classified as strong, deep in the inertial range magnetic field lines are nearly parallel across perpendicular separations $\lambda_{\perp}$ and nearly straight along parallel separations $\lambda_{\|}$; differential bending angles are of order $\left(\lambda_{\perp} / L\right)^{1 / 3} \sim\left(\lambda_{\|} / L\right)^{1 / 2}$.

\subsection{Parallel cascade}

It is interesting to examine the frequency changing interactions that drive the parallel cascade. Referring back to the intermediate cascade, we know that 3-wave interactions do not change frequencies. However, interactions involving more than 3 -waves can. For example, frequency changes arise in 4-wave interactions of the form

$$
\begin{gathered}
\omega_{1}+\omega_{2}+\omega_{3}=\omega_{4}, \\
\mathbf{k}_{1}+\mathbf{k}_{2}+\mathbf{k}_{3}=\mathbf{k}_{4},
\end{gathered}
$$

** This assumption is motivated by the expectation that the excitation of interstellar turbulence is characteristically strong. 


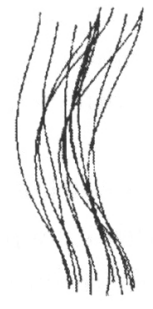

a. Field line wander

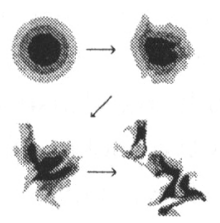

b. Wave packet distortion

Figure 1. The left hand panel displays a sample of field lines perturbed by downward propagating waves. The distortion of an originally circular bullseye pattern as it moves upward following these field lines is shown in the right hand panel.

where $k_{1 z}$ and $k_{2 z}$ have the same sign and $\omega_{3}=v_{A}\left|k_{3 z}\right|=0(\mathrm{Ng}$ and Bhattacharjee, 1996; GKII). The parallel cascade they give rise to proceeds at a rate which is smaller than that of the perpendicular cascade by a factor of order $\chi$. Because strong MHD turbulence is characterized by $\chi \sim 1$, it has a significant parallel cascade.

\subsection{FIELD LINE GEOMETRY}

MHD turbulence is best understood geometrically. Field lines perturbed by waves propagating in one direction define 2-dimensional mappings between $x y$ planes separated by distance $z$. Shear Alfvén waves dominate the shear and slow waves the dilatation of these mappings. The magnitude of the shear exceeds that of the dilatation by a factor of order $\lambda_{\|} / \lambda_{\perp} \sim\left(L / \lambda_{\perp}\right)^{1 / 3} \gg 1$. These mappings describe the distortion that counterpropagating waves would suffer if they moved at uniform speed along the perturbed field lines. The dominance of the shear over the dilatation explains why shear Alfvén waves control the perpendicular cascades of both types of wave.

The recognition that MHD waves tend to follow field lines is essential to understanding their turbulent cascades. Figure 1 provides a visual illustration of how this works. The left hand panel displays a snapshot of field lines perturbed by downward propagating waves. In the right hand panel we follow the evolution of a horizontal pattern as it propagates from the bottom to the top following these lines. The distortion of the initially circular bullseye is principally due to the shear in the two dimensional mapping defined by the perturbed field lines. The cascade time on the scale of the initial pattern is that over which the shear grows to order unity.

This geometrical picture requires two qualification. The first is that the propagation speed of MHD waves is not exactly constant but varies with the strength of the local magnetic field. Pressure perturbations associated with slow waves are balanced by perturbations of magnetic pressure. The resulting perturbations in propagation speed, of order $v_{\lambda_{\perp}}$, contribute to the nonlinear cascade. Over one 
wave period they lead to fractional distortions of order $v_{\lambda_{\perp}} / v_{A} \sim \lambda_{\perp} / \lambda_{\|} \ll 1$. Thus they are properly ignored. The second qualification is that MHD waves do not exactly follow field lines. The extent to which this effects their cascade remains to be quantified.

The parallel cascade may also be viewed in geometrical terms. Consider an upward propagating wave packet of length $\lambda_{\|}$and width $\lambda_{\perp}$ which is being distorted by downward moving wave packets of similar scale. Correlations along the parallel direction are shortened because the front and back of the wave packet undergo different 2-dimensional mappings. This happens because the upward propagating packet distorts each downward going packet as it passes through it. This distortion is of order $\chi$. For strong MHD turbulence $\chi \sim 1$ which accounts for its significant parallel cascade.

Incidentally, the geometrical picture also aids the interpretion of results from perturbation theory. For example, the 3 -wave resonant interactions which dominate the perpendicular cascade and the 4-wave resonant interactions which cause the lowest order frequency changes each depend upon the amplitudes of modes with $k_{z}=0$. This is because the shear in the mapping between $x y$ planes separated by $\Delta z$ is proportional to the displacement amplitudes of modes with $k_{z} \lesssim 1 / \Delta z$. Perturbation theory corresponds to the limit of vanishing cascade strength in which shears of order unity are achieved in the limit of infinite separation along the $z$-axis.

\section{Application To Interstellar Scattering}

My contribution is not intended to cover astronomical applications. Yoram Lithwick's presentation describes the relation of MHD turbulence to ISS. However, since his talk is not written up, I'll make a few relevant comments.

Most of the baryonic matter in the universe has such high electrical conductivity that magnetic fields diffuse very slowly through it. Thus fluid motions and motions of magnetic field lines are closely coupled. Large scale fluid motions are generally turbulent, and incompressible MHD is the simplest approximation under which we can study these complex coupled motions. But why should astronomers be concerned with the inertial range of MHD turbulence? Motions on the largest scale, the energy bearing eddies, dominate the transport of momentum and energy. Inertial range eddies play only a minor role in transport. Below I offer one example with the inertial range of interstellar turbulence is relevant to observations.

The spectrum of interstellar electron density fluctuations probably reveals an underlying turbulent cascade. Elliptical, scatter-broadened images imply the density spectrum is anisotropic, presumable due to the presence of a large scale magnetic field. Shear Alfvén waves, which control the cascade, cannot be directly responsible for density fluctuations since they do not produce pressure perturbations. However, there is ample reason to believe that they passively mix both specific entropy and slow waves, and that this forces the power spectra of these 
fields to ape that of the shear Alfvén waves. Density fluctuations might then be due to either isobaric fluctuations of specific entropy or to pressure fluctuations associated with slow waves. The former has a close analogy in the Earth's atmosphere where specific entropy mixed by hydrodynamic turbulence gives rise to the refractivity variations that cause optical scintillations. Each of these proposals for generating density fluctuations suffers from unresolved difficulties which are briefly described the following paragraph.

The bulk of the ionized interstellar gas is found in HII regions. Thus it is natural to look to these as the prime sites for ISS. However, specific entropy is not conserved during large scale motions in HII regions. Thermal relaxation of the gas temperature occurs on much shorter timescales than these motions. Only small scale motions conserve entropy. This poses a serious problem for matching the magnitude of the ISS with entropy fluctuations. Slow waves might appear to provide a better solution. But they are strongly damped in collisionless plasmas, and the ionized gas in HII regions is effectively collisionless on the scales implicated in diffractive scintillations. There are plausible resolutions to each of these difficulties, but they lie outside the scope of this paper.

\section{Acknowledgements}

The research reported here was carried out in collaboration with S. Sridhar. I am grateful to J. Maron for supplying me with Figure 1. Financial support was provided by the National Science Foundation of the United States.

\section{References}

Goldreich P. and Sridhar S.: 1995, Toward a Theory of Interstellar Turbulence. II. Strong Alfvénic Turbulence, Astrophys. J. 438, 763-775.

Goldreich P. and Sridhar S.: 1997, Magnetohydrodynamic Turbulence Revisited, Astrophys. J. 485, 680-688.

Iroshnikov, P.S.: 1963, Turbulence of a Conducting Fluid in a Strong Magnetic Field, Astronomicheskii Zhurnal 40, 742-750.

Kraichnan, R.H.: 1965, Inertial Range Spectrum of Hydromagnetic Turbulence, Physics of Fluids $\mathbf{8}$, 1385-1387.

Ng, C.S. and Bhattacharjee, A.: 1996, Interaction of Shear-Alfven Wave Packets: Implication for Weak Magnetohydrodynamic Turbulence in Astrophysical Plasmas, Astrophys. J. 465, 845-854.

Shebalin, J.V., Matthaeus, W.H. and Montgomery, D.: 1983, Anisotropy in MHD Turbulence Due to a Mean Magnetic Field, J. Plasma Phys. 29, 525-547. 